\title{
Study on Fire Protection Design of Large Shopping Centers
}

\author{
Zhang Qian \\ The Ministry of Scientific Research, Training Department, Public Security Fire Brigade Junior \\ College, Kunming, Yunnan Province 650208
}

Keywords: Large shopping centers; fire scenes; fire protection; evacuation

\begin{abstract}
Fires occurring in comparatively closed places like large shopping centers where evacuation is quite inconvenient make great loss, including casualties and destroys of materials. It not only makes people fall into panic, influencing their life, but results in a great economic loss. In the past few years, lots of scholars had done some researches on fire protection. Most of them analyzed the characteristics of fires occurred in large shopping centers, did an assessment, and gave some suggestions on fire protection and evacuation. Similarly, this dissertation aims at reducing casualties in fires and protecting social stabilization through the study on fire protection assessment. This paper intends to analyze the characteristics of fires in large shopping centers, make fire scenes, find appropriate and safe exit to make a good plan for personnel evacuation. Characteristics of fires in large shopping centers will be introduced in the first part in the main body. The most important part lies in finding safe exit so as to get a better evacuation. The conclusion is that large shopping centers where are often crowded, need to be paid more attention to fire protection. Through the fire protection, losses caused by fires occurred in the shopping centers can be reduced and it helps a lot in boosting prominently the shopping centers' economic and social effect.
\end{abstract}

\section{Importance of Fire Protection in Large Shopping Centers}

In general, consumers and service staffs in large shopping centers are always in a large number. And the more people in the shopping centers are, the more electricity consumption will be. On such occasion, the faster the building will be destroyed, even some accidents like fires will occur. Once the fire occurred, people will fall into panic immediately. They will be hurried in escaping, and then treads accident will possibly happen. In China, fires occurring in large shopping centers always make great destruction, as figure 1 shows in the following. The black one indicates the direct property loss and the other shows the number of casualties in the fire. We can see, the loss is beyond imagination. Consequently, fire protection in large shopping centers is supposed to arouse enough concern. It needs to be paid attention not only to fire avoidance but also personnel evacuation.

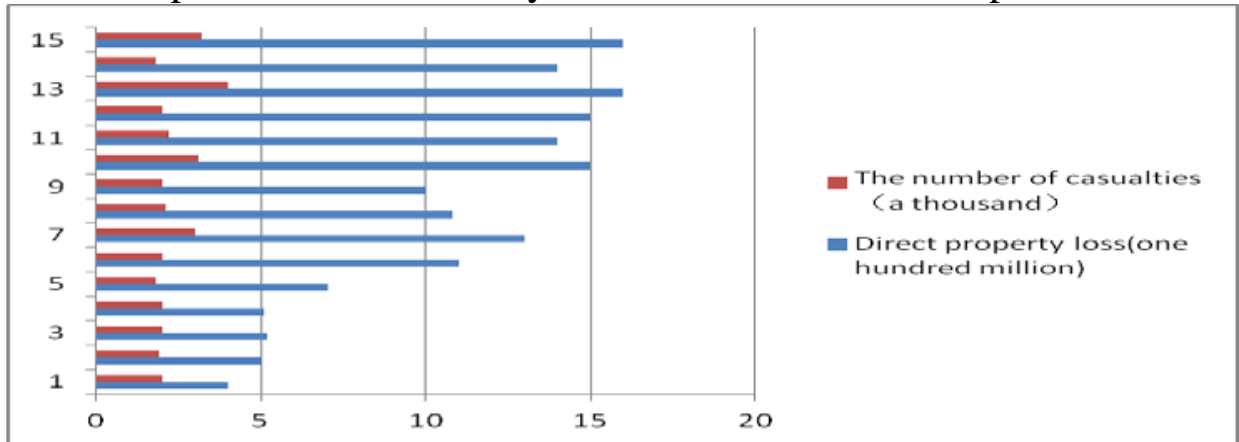

Figure 1 Direct loss of and casualties in fires from 1988 to 2002

\section{Characteristics of Fires in Large Shopping Centers}

Compared to fires in open areas like forest, fires in large shopping centers have more obstacles in personnel evacuation because of the closed circumstance. In order to make a good plan for fire protection in large shopping centers, knowing the general characteristics of fires in such kind of places is absolutely necessary. 


\subsection{Poor Ventilation and High Temperature}

In large shopping centers, there are always seldom windows and the exits are few. As a consequence, the ventilation is quite poor. Once fires occur, some inflamers will release smokes, and then people will feel lack of oxygen. In addition, the temperature of large buildings above ground can reach eight hundred degree or so. Effected by irritant even poisonous essence in the smokes, people will possibly die within one minute.

\subsection{Various Combustible Articles}

Articles in large shopping centers are of variety, and some of them are combustible, like clothes, lighter and so on. All of these articles are likely to become fire source. What's worse, once fire occurred, clothes, and fabric products will make it more serious, which increases the fire load of those shopping centers. Once larger the fire load becomes and longer the building combusts, collapse is possibly happen. In addition, gases released from combustible building and ornament materials are generally poisonous. It directly brings about that people get poisoned. This is undoubtedly another big threat to people’s safety.

\section{Fire Protection and Assessment}

As showed by previous researches that fires have become such a big threat to people's safety, actions have to be taken without any delay. A large shopping center is taken as an example to do the research on fire protection and assessment. It has two floors with height in 11 meters, plane size of 80 meters by 110 meters, floor space of 11100 square meters, and the number of total staffs is 173 . The first floor is used as a supermarket, mainly putting foods and daily-use sundries. While the second is taken as commercial area and the main products are clothes, shoes, bags and suitcases. Since the floor space of this shopping center is large and combustible articles are various, and it is located in the center of the city that the number of consumers is large, it would be a typical and representative one in fire protection and assessment.

\subsection{Setting Fire Source}

In this shopping center, combustible articles are mostly seen in clothes in the second floor. Clothes are easily to be combusted and heat released is huge so that it is characterised by rapid in combustible speed and high in heat release rate. In consequence, according to the distribution of combustible articles in the shopping center and the principle of choosing the most disadvantages place in fire source setting, clothes area in the second floor would be the best choice as fire source.

\subsection{Confirming Heat Release Rate}

Most of fires occurring in the civil buildings spread slowly in the very beginning, while they speed up when they get to a certain degree. A previous research shows that the heat release rate is in direct propotion to the square of fire spreading time. In general, the value of fire spreading coefficient has four kinds, slow fire, medium fire, rapid fire, and ultrafast fire. And they will get to a fire scale of one MW when the fire spreading time reaches 600 seconds, 300 seconds, 150 seconds, and 75 seconds respectively. It is clearly showed as follows.

Table 1 Fire spreading coefficient

\begin{tabular}{llcc}
\hline Classifications of fires & Combustible materials & Fire spreading coefficient(kW/s $)$ & Time(s) \\
\hline Slow fire & Furniture in hardwood & 0.00293 & 600 \\
Medium fire & Cotton or polyester cushion & 0.01172 & 300 \\
Rapid fire & Mail bags and wooden shelves & 0.04689 & 150 \\
Ultrafast fire & Light curtains & 0.1875 & 75
\end{tabular}

It has been proved that the factors for fire spreading coefficient is difficult to confirm. While Tianjin fire control research laboratory did a clothes fire test and it showed that heat release rate of those clothes stacked horizontally is smaller than that of medium fire. Comparatively, heat release rate of those pensile clothes is bigger than that of medium fire. Since fire caused by clothes burning 
is almost the most dangerous among all the other kinds of fires and the clothes are always pensile or stacking, the fire is likely to spread in rapid speed once it occurred. That is to say, heat release rate equals to $0.04689 \mathrm{~kW} / \mathrm{s}^{2}$.

\subsection{Assessing the Time}

The objective of assessing is to make sure whether the time from fire occurring to dangerous situation is longer than the time from fire occurring to people's safe evacuation or not. If it is longer, the fire protection is successful and people will be safe. On the contrary, if it is shorter, the fire protection is proved to be failing and the number of casualties will be larger.

People's safe evacuation time generally includes alarming time, responding time, and people's moving time. Since the space of shopping centers is large, the alarming time may be different due to the distance from people's location to fire source. Those who are near to the fire source may alarm fire firstly, while those who are far away from fire source can only know fire occurring by broadcasting. Therefore, by calculating, the average alarming time is about 90 seconds. Responding time means time from awareness of fire to evacuation's beginning. It is influenced by many factors, including different building places, fire alarm system, and people's experience in fire. It is estimated about 45 seconds on average. People's moving time is also of variety. The old and children always moves slowly because of their physical quality, while the young moves fast. So the average moving time is caculated as sixty meters per minutes, about 182 seconds. That's to say, the total safe evacuation time is 317 seconds, about five minutes. In a word, if people can alarm quickly and move fast, get away from the fire source in five minutes, then they will be safe.

\section{Conclusion}

By setting fire source, confirming heat release rate, and assessing the time, matters need to be paid attetion to in fires occurring in large shopping centers are known. As long as people can evacuate within the total safe evacuation time, they will be safe. For shortening the evacuation time, people need to know more information about self-rescue in fires and give an alarm to other people at once. In addition, in the places where combustible articles are various, more fire alarming system is necessary to be set up. What's more, in large shopping centers, exits are supposed to be set as many as possible and mark of their location needs to be clear. In conclusion, fire protection for large shopping centers where the number of people is large is necessary to be paid enough attention to. Fire protection design of buildings and facilities is a good breach. Information about fire protection and self-rescue in fires is supposed to be widely spread.

\section{Reference}

[1] Kevin McGrattan, Glenn Forney. Fire D raamies Simulator (Version 4) Technical Reference Guide [M]. Washingto: U.S. governin g printing oftice, 2004.

[2] Lei Bai, Zengfeng Yan, Shuping Zhang. The Application of A New Digital Method for Fire Protection Design in The Large Commercial Building Field [A]. School of Architecture, Xi'an University of Architecture and Technology, 2011.

[3] Li Haikun. The Reserch of Anhui Ancient Building Fire Protection System [A]. Department of Mechanical and Electrical Engineering, Anhui University of Architecture, 2012.

[4] David Rush, Luke Bisby, Allan Jowsey. Evaluating Design Guidance for Intumescent fire protection of Concrete Filled Steel Hollow Sections [A]. BRE Centre for Fire Safety Engineering,University of Edinburgh, 2014.

[5] Ding Pei, Zou Yin, Deng Xiaoyang. Study on Fire Protection and Design of Large Commercial buildings[D]. Tianjin University, 2012.

[6] Ma Qianli, Huang Tinglin, Ni Zhaopeng. Study on Comprehensive Fire Protection Method of Large Commercial Buildings[D]. Xi'an University of Architecture and Technology, 2011. 
[7] Syed Shahid Ali. Fire Protection Service in Karachi[J]. Journal of Basic \& Applied Sciences, 2015(11):176.

[8] Zehfuss J. Innovations are Needed in Fire Protection[A]. Web of Science. ERNST \& SOHN, 2015(5): 321-322. 